\title{
THERMAL DEFLECTIONS OF ANISOTROPIC THIN PLATES*
}

\author{
BY \\ WILLIAM H. PELL** \\ Bell Aircraft Corporation
}

1. Introduction. Equations governing the deflection of an isotropic thin elastic plate subjected to a temperature distribution of the form

$$
T(x, y, z)=T_{0}(x, y)+z T_{1}(x, y),
$$

where the neutral plane of the plate is taken to lie in the $x y$-plane, have been derived by Nádai. ${ }^{1}$ He did not consider the solution of these equations, and they have not been treated to any considerable extent by subsequent writers in thermo-elasticity.2 The isothermal theory of anisotropic thin elastic plates has been developed principally by Boussinesq, ${ }^{3}$ Voigt, ${ }^{4}$ and Lechnitzky. ${ }^{5}$ It appears that the only treatment of thermal effects for the anisotropic plate is due to Voigt, ${ }^{6}$ who considers a simple case in which no bending of the plate occurs.

The first part of this paper is concerned with the derivation of two partial differential equations governing the deflection of a thin elastic plate possessing one plane of elastic symmetry parallel to the faces of the plate, and subjected to a temperature distribution described by a function of the form (1.1). One of these equations, with suitable boundary conditions, defines a stress function $F$; the other, the deflection function $w$. In the second part, recent results in anisotropic plate theory are used to solve the equation for the stress function with rather general boundary conditions for the case where $T_{0}(x, y)$ is a polynomial in $x$ and $y$. The problem of solving the equation of the deflection is a difficult one, and a solution valid throughout the region enclosed by the plate is not available. Since the thermal deflection problem for the isotropic plate is of interest in itself, however, the case of the isotropic circular plate with radial temperature distribution is considered in the concluding portion, and the solution is obtained.

2. The thermo-elastic equations for anisotropic plates. Let us consider a thin elastic plate composed of a medium possessing at each point at least one plane of elastic symmetry parallel to the middle plane of the plate, which is chosen to lie in the $x y$-plane. Let the plate be subjected in its interior to a temperature distribution given by (1.1). The plate is supposed acted upon by forces on its edge lying in the middle plane, but to be free of lateral load and body forces.

* Received August 10, 1945.

** This paper was prepared under the direction of Professor I. S. Sokolnikoff, whose valuable assistance and helpful suggestions are gratefully acknowledged. At the time of writing, the author was a Fellow under the Program of Advanced Instruction and Research in Mechanics at Brown University.

1 A. Nádai, Elastische Platten, Springer, Berlin, 1925, pp. 264-268.

${ }^{2}$ J. N. Goodier, Physics 7, 156-159 (1936); N. Yamaguti, J. Fac. Eng., Tokyo Imperial University 28, no. 1 (1928).

M. J. Boussinesq, J. de Math. (Liouville) (3) 5, 359 (1879).

'W. Voigt, Lehrbuch der Kristallphysik, Teubner, Leipzig and Berlin, 1910, pp. 675-698, pp. 763-791,

${ }^{5}$ S. G. Lechnitzky, App. Math. Mech., Leningrad, 3, old series 56-69 (1936), new series 1, 77-90 (1939); new series 2, 185-209 (1938).

- W. Voigt, loc. cit., \$388, ch. 7 . 
The Kirchhoff assumptions of the thin plate theory lead to the well-known fundamental relations

$$
\begin{gathered}
u=-z \frac{\partial w}{\partial x}, \quad v=-z \frac{\partial w}{\partial y}, \\
\left|\tau_{z z}\right| \ll \max \left\{\left|\tau_{x x}\right|,\left|\tau_{y y}\right|,\left|\tau_{x y}\right|\right\},
\end{gathered}
$$

valid throughout the thickness, $2 h$, of the plate, where $w$ is the deflection of the middle surface of the plate, and $u$ and $v$ are the displacements of a point $(x, y, z)$ of the plate in the $x$ - and $y$-directions, respectively. To the assumptions (2.1) and (2.2) is adjoined the following one: the stress tensor $\tau$ at any point in the plate is the sum

$$
\tau=\tau^{0}+\tau^{1}
$$

where $\tau^{0}$ is a plane stress tensor generated by $T_{0}(x, y)$ and reactions at the plate edge. and $\tau^{1}$ arises from the bending of the plate, i.e., from the action of $z T_{1}$. It should be remarked that this supposition is fundamental in the thermo-elastic theory of thin plates presented here. Together with (2.1) and (2.2) it serves here the same purpose that (2.1) and (2.2) do alone in the isothermal theory, i.e., they reduce the thermoelastic plate problem to one two-dimensional in character.

The generalized Hooke's law ${ }^{7}$ taking into account thermal effects is expressed by

$$
\begin{aligned}
\frac{\partial u}{\partial x} & =a_{11} \tau_{x x}+a_{12} \tau_{y y}+a_{13} \tau_{z z}+a_{16} \tau_{x y}+a_{1} T, \\
\frac{\partial v}{\partial y} & =a_{12} \tau_{x x}+a_{22} \tau_{y y}+a_{23} \tau_{z z}+a_{26} \tau_{x y}+a_{2} T, \\
\frac{\partial w}{\partial z} & =a_{13} \tau_{x x}+a_{23} \tau_{y y}+a_{33} \tau_{z z}+a_{36} \tau_{x y}+a_{3} T, \\
\frac{\partial v}{\partial z}+\frac{\partial w}{\partial y} & =a_{44} \tau_{y z}+a_{45} \tau_{x z}+2 a_{4} T, \\
\frac{\partial w}{\partial x}+\frac{\partial u}{\partial z} & =a_{45} \tau_{y z}+a_{55} \tau_{x z}+2 a_{5} T, \\
\frac{\partial u}{\partial y}+\frac{\partial v}{\partial x} & =a_{16} \tau_{x x}+a_{26} \tau_{y y}+g_{36} \tau_{z z}+a_{66} \tau_{x y}+2 a_{6} T,
\end{aligned}
$$

where the $a_{i j}$ are elastic constants and $a_{i}$ are the coefficients of thermal expansion of thermal expansion of the medium. For the plane stress system ${ }^{8}(2.4)$ reduces to

$$
\begin{aligned}
\frac{\partial u^{0}}{\partial x} & =a_{11} \tau_{x x}^{0}+a_{12} \tau_{y y}^{0}+a_{16} \tau_{x y}^{0}+a_{1} T_{0}, \\
\frac{\partial v^{0}}{\partial y} & =a_{12} \tau_{x x}^{0}+a_{22} \tau_{y y}^{0}+a_{26} \tau_{x y}^{0}+a_{2} T_{0}, \\
\frac{\partial u^{0}}{\partial y}+\frac{\partial v^{0}}{\partial x} & =a_{16} \tau_{x x}^{0}+a_{26} \tau_{y y}^{0}+a_{66} \tau_{x y}^{0}+2 a_{6} T_{0} .
\end{aligned}
$$

7 A. E. H. Love, Mathematical theory of elasticity, Cambridge University Press, Cambridge, ed. 4, 1927, pp. 151-160.

${ }_{8}$ Displacements, strains, and stresses associated with $T_{0}$ will be denoted by the superscript 0 ; those associated with $z T_{1}$, by the superscript 1 . 
With the introduction of a stress function $F(x, y)$, one has

$$
\tau_{x x}^{0}=\frac{\partial^{2} F}{\partial y^{2}}, \quad \tau_{x y}^{0}=-\frac{\partial^{2} F}{\partial x \partial y}, \quad \tau_{y y}^{0}=\frac{\partial^{2} F}{\partial x^{2}} .
$$

The resultants of these stresses acting across the thickness of the plate are, respectively,

$$
N_{x x}=2 h \tau_{x x}^{0}, \quad N_{x y}=2 h \tau_{x y}^{0}, \quad N_{y y}=2 h \tau_{y y}^{0} .
$$

Since the displacements must satisfy a compatibility condition, it follows from (2.5) and (2.6) that $F$ must satisfy the equation

$$
\begin{aligned}
a_{22} \frac{\partial^{4} F}{\partial x^{4}}-2 a_{26} \frac{\partial^{4} F}{\partial x^{3} \partial y}+\left(2 a_{12}+a_{66}\right) & \frac{\partial^{4} F}{\partial x^{2} \partial y^{2}}-2 a_{16} \frac{\partial^{4} F}{\partial x \partial y^{3}}+a_{11} \frac{\partial^{4} F}{\partial y^{4}} \\
& =-\left\{a_{2} \frac{\partial^{2} T_{0}}{\partial x^{2}}-2 a_{6} \frac{\partial^{2} T_{0}}{\partial x \partial y}+a_{1} \frac{\partial^{2} T_{0}}{\partial y^{2}}\right\} .
\end{aligned}
$$

It is assumed that the derivatives appearing in (2.7) are continuous.

The generalized Hooke's law for the strains and stresses associated with the temperature function $z T_{1}$ permits one to write

$$
\begin{aligned}
\tau_{x x}^{1}= & c_{11}\left(\frac{\partial u^{1}}{\partial x}-a_{1} z T_{1}\right)+c_{12}\left(\frac{\partial v_{1}}{\partial y}-a_{2} z T_{1}\right)+c_{13}\left(\frac{\partial w^{1}}{\partial z}-a_{3} z T_{1}\right) \\
& +c_{16}\left(\frac{1}{2}\left[\frac{\partial u^{1}}{\partial y}+\frac{\partial v_{1}}{\partial x}\right]-a_{6} z T_{1}\right), \\
\tau_{y y}^{1}= & c_{12}\left(\frac{\partial u^{1}}{\partial x}-a_{1} z T_{1}\right)+c_{22}\left(\frac{\partial v^{1}}{\partial y}-a_{2} z T_{1}\right)+c_{23}\left(\frac{\partial w^{1}}{\partial z}-a_{3} z T_{1}\right) \\
& +c_{26}\left(\frac{1}{2}\left[\frac{\partial u^{1}}{\partial y}+\frac{\partial v^{1}}{\partial x}\right]-a_{6} z T_{1}\right), \\
\tau_{z z}^{1}= & c_{13}\left(\frac{\partial u^{1}}{\partial x}-a_{1} z T_{1}\right)+c_{23}\left(\frac{\partial v^{1}}{\partial y}-a_{2} z T_{1}\right)+c_{33}\left(\frac{\partial w^{1}}{\partial z}-a_{3} z T_{1}\right) \\
& +c_{36}\left(\frac{1}{2}\left[\frac{\partial u^{1}}{\partial y}+\frac{\partial v^{1}}{\partial x}\right]-a_{6} z T_{1}\right), \\
\tau_{x y}^{1}= & c_{16}\left(\frac{\partial u^{1}}{\partial x}-a_{1} z T_{1}\right)+c_{26}\left(\frac{\partial v^{1}}{\partial y}-a_{2} z T_{1}\right)+c_{36}\left(\frac{\partial w^{1}}{\partial z}-a_{3} z T_{1}\right) \\
& +c_{66}\left(\frac{1}{2}\left[\frac{\partial u^{1}}{\partial y}+\frac{\partial v^{1}}{\partial x}\right]-a_{6} z T_{1}\right) .
\end{aligned}
$$

The assumption (2.1) gives

$$
\frac{\partial u^{1}}{\partial x}=-z \frac{\partial^{2} w}{\partial x^{2}}, \quad \frac{\partial v^{1}}{\partial y}=-z \frac{\partial^{2} w}{\partial y^{2}}, \quad \frac{1}{2}\left(\frac{\partial u^{1}}{\partial y}+\frac{\partial v^{1}}{\partial x}\right)=-z \frac{\partial^{2} w}{\partial x \partial y},
$$


and (2.9) applied to the third equation of (2.8) yields

$$
\begin{aligned}
\frac{\partial w}{\partial z}-a_{3} z T_{1}=- & \frac{z}{c_{33}}\left\{c_{13}\left(\frac{\partial^{2} w}{\partial x^{2}}-a_{1} T_{1}\right)+c_{23}\left(\frac{\partial^{2} w}{\partial y^{2}}-a_{2} T_{1}\right)\right. \\
& \left.+c_{36}\left(\frac{\partial^{2} w}{\partial x \partial y}-a_{6} T_{1}\right)\right\} .
\end{aligned}
$$

Now (2.9) and (2.10) are inserted in (2.8) with the result

$$
\begin{aligned}
& \tau_{x x}^{1}=-z\left(b_{11} \frac{\partial^{2} w}{\partial x^{2}}+b_{12} \frac{\partial^{2} w}{\partial y^{2}}+b_{16} \frac{\partial^{2} w}{\partial x \partial y}+\alpha_{1} T_{1}\right), \\
& \tau_{y y}^{1}=-z\left(b_{12} \frac{\partial^{2} w}{\partial x^{2}}+b_{22} \frac{\partial^{2} w}{\partial y^{2}}+b_{26} \frac{\partial^{2} w}{\partial x \partial y}+\alpha_{2} T_{1}\right), \\
& \tau_{x y}^{1}=-z\left(b_{16} \frac{\partial^{2} w}{\partial x^{2}}+b_{26} \frac{\partial^{2} w}{\partial y^{2}}+b_{66} \frac{\partial^{2} w}{\partial x \partial y}+\alpha_{6} T_{1}\right),
\end{aligned}
$$

where

$$
\begin{aligned}
b_{i k} & =c_{i k}-\frac{c_{i 3} c_{k 3}}{c_{33}}, & i, k & =1,2,6, \\
\alpha_{i} & =b_{1 i} a_{1}+b_{2 i} a_{2}+b_{6 i} a_{6}, & i & =1,2,6 .
\end{aligned}
$$

Noting $\left.\left.\tau_{y z}^{1}\right]_{z= \pm h}=\tau_{x z}^{1}\right]_{z= \pm h}=0$, we may now integrate, with respect to $z$, the equations of equilibrium

$$
\frac{\partial \tau_{x x}^{1}}{\partial x}+\frac{\partial \tau_{x y}^{1}}{\partial y}+\frac{\partial \tau_{x z}^{1}}{\partial z}=0, \quad \frac{\partial \tau_{x y}^{1}}{\partial x}+\frac{\partial \tau_{y y}^{1}}{\partial y}+\frac{\partial \tau_{y z}^{1}}{\partial z}=0,
$$

obtaining

$$
\begin{aligned}
\tau_{x z}^{1}= & \frac{z^{2}-h^{2}}{2}\left[b_{11} \frac{\partial^{3} w}{\partial x^{3}}+2 b_{16} \frac{\partial^{3} w}{\partial x^{2} \partial y}+\left(b_{12}+b_{66}\right) \frac{\partial^{3} w}{\partial x \partial y^{2}}\right. \\
& \left.+b_{26} \frac{\partial^{3} w}{\partial y^{3}}+\alpha_{1} \frac{\partial T_{1}}{\partial x}+\alpha_{6} \frac{\partial T_{1}}{\partial y}\right], \\
\tau_{y z}^{1}= & \frac{z^{2}-h^{2}}{2}\left[b_{16} \frac{\partial^{3} w}{\partial x^{3}}+\left(b_{12}+b_{66}\right) \frac{\partial^{3} w}{\partial x^{2} \partial y}+2 b_{26} \frac{\partial^{3} w}{\partial x \partial y^{2}}\right. \\
& \left.+b_{22} \frac{\partial^{3} w}{\partial y^{3}}+\alpha_{6} \frac{\partial T_{1}}{\partial x}+\alpha_{2} \frac{\partial T_{1}}{\partial y}\right] .
\end{aligned}
$$

The resultants of these stresses acting across the thickness of the plate are, respectively,

$$
Q_{x}=\int_{-h}^{h} \tau_{x z} d z, \quad Q_{y}=\int_{-h}^{h} \tau_{y x} d z .
$$

The following equation expressing the condition of statical equilibrium of an arbi- 
trary element of the plate may be obtained in the usual way ${ }^{9}$ since the derivation does not depend on the material composing the plate:

$$
\frac{\partial Q_{x}}{\partial x}+\frac{\partial Q_{y}}{\partial y}+N_{x x} \frac{\partial^{2} w}{\partial x^{2}}+2 N_{x y} \frac{\partial^{2} w}{\partial x \partial y}+N_{y y} \frac{\partial^{2} w}{\partial y^{2}}=0 .
$$

The values of $Q_{x}$ and $Q_{y}$ obtained from (2.12) are now introduced, and the result is the following differential equation for the deflection:

$$
\begin{aligned}
b_{11} \frac{\partial^{4} w}{\partial x^{4}}+3 b_{16} \frac{\partial^{4} w}{\partial x^{3} \partial y}+2\left(b_{12}\right. & \left.+b_{66}\right) \frac{\partial^{4} w}{\partial x^{2} \partial y^{2}}+3 b_{26} \frac{\partial^{4} w}{\partial x \partial y^{4}}+b_{22} \frac{\partial^{4} w}{\partial y^{4}} \\
= & -\left\{\alpha_{1} \frac{\partial^{2} T_{1}}{\partial x^{2}}+2 \alpha_{6} \frac{\partial^{2} T_{1}}{\partial x \partial y}+\alpha_{2} \frac{\partial^{2} T_{1}}{\partial y^{2}}\right\} \\
& +\frac{3}{2 h^{3}}\left\{N_{x x} \frac{\partial^{2} w}{\partial x^{2}}+2 N_{x y} \frac{\partial^{2} w}{\partial x \partial y}+N_{y y} \frac{\partial^{2} w}{\partial y^{2}}\right\} .
\end{aligned}
$$

Again, the continuity of the derivatives appearing is assumed.

The portion of the $x y$-plane occupied by the middle plane of the plate will be called $S_{0}$; the boundary of $S_{0}$ will be called $C_{0}$, and it will be assumed that $C_{0}$ is an analytic curve. The problem of thermo-elastic deflection is solved if a solution for each of (2.7) and (2.13) valid throughout $S_{0}$ can be found which satisfies appropriate boundary conditions on $C_{0}$.

3. The stress function. Since derivatives of $F$ appear in (2.13), the solution of (2.7) will be considered first. Lechnitzky ${ }^{10}$ has shown that the roots of the characteristic equation

$$
a_{11} \mu^{4}-2 a_{16 \mu^{3}}+\left(2 a_{12}+a_{66}\right) \mu^{2}-2 a_{26 \mu}+a_{22}=0
$$

are necessarily complex. These roots will be denoted by $\mu_{k}=\alpha_{k} \pm i \beta_{k}, k=1,2$, where $\beta_{k} \neq 0$. Two cases must be distinguished: $\mu_{1} \neq \mu_{2}$ and $\mu_{1}=\mu_{2}$.

If $F_{p}$ denotes a particular solution, and $\mu_{1}$ and $\mu_{2}$ are distinct, then the most general solution of (2.7) is given by

$$
F(x, y)=F_{1}\left(z_{1}\right)+\bar{F}_{1}\left(\bar{z}_{1}\right)+F_{2}\left(z_{2}\right)+\bar{F}_{2}\left(\bar{z}_{2}\right)+F_{p}(x, y),
$$

where $F_{k}\left(z_{k}\right)$ is in each case an arbitrary function of $z_{k}=x+\mu_{k} y$. The $F_{k}\left(z_{k}\right)$ is analytic in the region $S_{k}$ of the $z_{k}$-plane which corresponds to the region $S_{0}$ of the $z$-plane under the transformation ${ }^{11}$

$$
z_{k}=p_{k} z+\bar{q}_{k} \bar{z}, \quad k=1,2,
$$

where

$$
p_{k}=\frac{1}{2}\left(1-i \mu_{k}\right), \quad q_{k}=\frac{1}{2}\left(1-i \bar{\mu}_{k}\right), \quad k=1,2 .
$$

If $\mu_{1}=\mu_{2}$, then

${ }^{9}$ A. Nádai, loc. cit., pp. 233-235.

${ }^{10} \mathrm{~S}$. G. Lechnitzky, loc. cit.

11 A summary of anisotropic plate theory in English can be found in I. S. Sokolnikoff's Mathematical theory of elasticity, mimeograph lecture notes, Brown University, Providence, R. I., 1941, pp. 319-329. 


$$
F(x, y)=\bar{z}_{1} F_{1}\left(z_{1}\right)+z_{1} \bar{F}_{1}\left(\bar{z}_{1}\right)+G_{1}\left(z_{1}\right)+\bar{G}_{1}\left(\bar{z}_{1}\right)+F_{p}(x, y) .
$$

For the plate without holes, $S_{k}$ is a simply-connected domain, and $F_{k}\left(z_{k}\right)$ and $G_{1}\left(z_{1}\right)$ are single-valued analytic functions in $S_{k}$ and $S_{1}$, respectively.

4. The boundary conditions for $F$. The plane stresses must satisfy the wellknown conditions

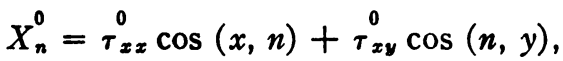

$$
\begin{aligned}
& Y_{n}^{0}=\tau_{x y}^{0} \cos (x, n)+\tau_{y y}^{0} \cos (y, n),
\end{aligned}
$$

where $X_{n}^{0}, Y_{n}^{0}$ are the $x$ - and $y$-components, respectively, of the force acting on the edge of the plate, and $n$ is the exterior unit normal to $C_{0}$. If $F$ is introduced through (2.6), the boundary conditions on $F$ may be written

$$
\frac{\partial F}{\partial x}+i \frac{\partial F}{\partial y}=i \int_{0}^{\cdot}\left(X_{n}^{0}+i Y_{n}^{0}\right) d s+c \equiv f_{1}(s)+i f_{2}(s)+c,
$$

where $s$ is the arc-length along $C_{0}$, measured from an arbitrary point with the usual convention as to positive $s$, and $c=c^{\prime}+i c^{\prime \prime}$ is an arbitrary, complex constant. A familiar alternative form of $(4.2)$ is

$$
\left.\begin{array}{rl}
\frac{\partial F}{\partial n} & =f(s), \\
F & =g(s),
\end{array}\right\} \text { on } C_{0},
$$

where $f$ and $g$ are prescribed functions along $C_{0}$, except for the arbitrary constants $c^{\prime}$ and $c^{\prime \prime}$ appearing in them.

The solutions considered here are assumed to be such that $F_{k}^{\prime}\left(z_{k}\right), F_{1}\left(z_{1}\right)$ and $G_{1}^{\prime}\left(z_{1}\right)$ are continuous in $S_{0}+C_{0}$. In this case (4.2) and (4.3) hold. To ensure the existence of such a solution, it is necessary to demand that

$$
\begin{gathered}
\int_{C_{0}} X_{n}^{0} d s=0, \quad \int_{C_{0}} Y_{n}^{0} d s=0, \\
\int_{C_{0}}\left\{f_{1}(s) \cos (x, s)+f_{2}(s) \cos (y, s)\right\} d s=0 .
\end{gathered}
$$

The physical significance of these conditions is of interest. Equation (4.4a) expresses mathematically the fact that the resultant of the external forces acting on the plate must vanish, and (4.4b) that the resultant of the external moments must vanish.

The analytical similarity between the boundary value problem presented by $(2.7)$ and (4.3) and that of the clamped plate under lateral load makes it possible to use recent results on the lattter problem obtained by Morkovin. ${ }^{12} \mathrm{His}$ treatment depends essentially on the handling of the boundary conditions.

The solution (3.2a) leads to boundary conditions expressed in terms of both $z_{1}$ and $z_{2}$, along either $C_{1}$ or $C_{2}$ (the boundaries of $S_{1}$ and $S_{2}$, respectively). Boundary conditions in terms of a single variable are obtained by mapping conformally a band of the $z_{k}$-plane containing $C_{k}$ in its interior onto an annular region of a $\zeta_{k}$-plane con-

12 V. Morkovin, Quart. Appl. Math. 1, 116-129 (1943). 
taining in its interior the circumference of the unit circle $\gamma_{k}$ in a way such that $C_{k}$ is mapped into $\gamma_{k}$. By a proper choice ${ }^{13}$ of the functions effecting this mapping, the transforms $z_{1}$ and $z_{2}$ of any given value of $z$ on $C_{0}$ can be mapped onto $\gamma_{1}$ and $\gamma_{2}$ in such a way that $\zeta_{1}=\zeta_{2}$. This common value is denoted by $\sigma=e^{i \phi}$. Thus the boundary conditions contain $\sigma$ alone.

Let

$$
z_{k}=\omega_{k}\left(\zeta_{k}\right), \quad k=1,2,
$$

be the functions achieving the desired mapping. Then $F$ has one of the forms

$$
\begin{aligned}
& F=\phi_{1}\left(\zeta_{1}\right)+\bar{\phi}_{1}\left(\bar{\zeta}_{1}\right)+\phi_{2}\left(\zeta_{2}\right)+\bar{\phi}_{2}\left(\bar{\zeta}_{2}\right)+F_{p}, \\
& F=\bar{\omega}_{1}\left(\bar{\zeta}_{1}\right) \phi_{1}\left(\zeta_{1}\right)+\omega_{1}\left(\zeta_{1}\right) \Phi_{1}\left(\bar{\zeta}_{1}\right)+\psi_{1}\left(\zeta_{1}\right)+\psi_{1}\left(\zeta_{1}\right)+F_{p},
\end{aligned}
$$

where $\phi_{k}\left(\zeta_{k}\right) \equiv F_{k}\left(\omega_{k}\left(\zeta_{k}\right)\right)$ and $\psi_{1}\left(\zeta_{1}\right) \equiv G_{1}\left(\omega_{1}\left(\zeta_{1}\right)\right)$. These functions are analytic and single-valued in some neighborhoods of $\gamma_{k}$, and hence possess Laurent expansions

$$
\phi_{k}\left(\zeta_{k}\right)=\sum_{n=-\infty}^{\infty} \gamma_{n k} \zeta_{k}^{n}, \quad \psi_{1}\left(\zeta_{1}\right)=\sum_{n=-\infty}^{\infty} \mu_{n 1} \zeta_{1}^{n} .
$$

The coefficients $\gamma_{n k}$ and $\mu_{n 1}$ are to be determined from the boundary conditions on $F$, expressed now on $\gamma_{1}$ or $\gamma_{2}$, as desired. If one defines

$$
\begin{aligned}
& H_{k}(\sigma)=p_{k} \omega_{k}^{\prime}(\sigma) \sigma+\bar{q}_{k} \bar{\omega}_{k}^{\prime}(\bar{\sigma}) \bar{\sigma}, \\
& J_{k}(\sigma)=\sigma\left(p_{k} \bar{p}_{k}+q_{k} \bar{q}_{k}\right)+\bar{\sigma} \frac{\bar{\omega}_{k}^{\prime}(\bar{\sigma})}{\omega_{k}^{\prime}(\sigma)} 2 p_{k} \bar{q}_{k},
\end{aligned}
$$

then boundary conditions on $\gamma_{1}$ or $\gamma_{2}(k=1$ or 2 , respectively) equivalent to (4.3) are given by

$$
\begin{aligned}
& \left.i \sigma \frac{d s}{d \sigma} \frac{\partial F}{\partial n}\right]_{C_{0}}=\frac{1}{2 \beta_{k}}\left[H_{k}(\sigma)\left(\frac{\partial F}{\partial x}-i \frac{\partial F}{\partial y}\right)_{c_{0}}+\bar{H}_{k}(\bar{\sigma})\left(\frac{\partial F}{\partial x}+i \frac{\partial F}{\partial y}\right)_{c_{0}}\right] \\
& F]_{C_{0}}=\frac{1}{2 \beta_{k}} \int_{1}^{\sigma}\left[H_{k}(\sigma)\left(\frac{\partial F}{\partial x}-i \frac{\partial F}{\partial y}\right)_{C_{0}}-\bar{H}_{k}(\bar{\sigma})\left(\frac{\partial F}{\partial x}+i \frac{\partial F}{\partial y}\right)_{c_{0}}\right] \frac{d \sigma}{\sigma}+c^{\prime \prime \prime},
\end{aligned}
$$

where $^{\mathrm{u}}$ the left-hand member of $(4.9 \mathrm{a})$ is defined by

$$
\left.\left.\left.i \sigma_{k} \frac{d s}{d \sigma_{k}} \frac{\partial F}{\partial n}\right]_{C_{0}}=\frac{1}{\beta_{k}}\left\{J_{k}\left(\sigma_{k}\right) \frac{\partial F}{\partial \sigma_{k}}\right]_{C_{0}}+\bar{J}_{k}\left(\bar{\sigma}_{k}\right) \frac{\partial F}{\partial \bar{\sigma}_{k}}\right]_{C_{0}}\right\}
$$

and $c^{\prime \prime \prime}$ is an arbitrary real constant.

5. The determination of $F$. The function $F$ will be determined under the assumption that the edge force vector (4.2) has the form ${ }^{15}$

$$
\left(\frac{\partial F}{\partial x}+i \frac{\partial F}{\partial y}\right)_{c_{0}}=\sum_{j=-k_{1}}^{k_{1}} c_{j} \sigma^{j}+c
$$

w V. Morkovin, loc. cit.

14 After the indicated differentiation of $\omega_{k}\left(\sigma_{k}\right), \sigma_{k}$ in $F$ and its derivative are to be replaced by the common value $\sigma$ of $\sigma_{1}$ and $\sigma_{2}$. Note also that $\bar{\sigma}=\sigma^{-1}$.

${ }^{15}$ Since $\sigma=e^{i \theta}$, it follows that $(\partial F / \partial x)_{C_{0}}$ and $(\partial F / \partial y)_{C_{0}}$ are trigonometric polynomials of order $k_{1}$. 
where the $c_{j}$ are complex constants. The temperature function $T_{0}(x, y)$ is taken to be the polynomial

$$
T_{0}(x, y)=\sum_{i=0}^{k} \sum_{j=0}^{i} t_{j, i-j}^{0} x^{j} y^{i-j},
$$

where the $t_{j, i-j}$ are real constants, and $k$ is an arbitrary integer greater than or equal to 0 . Then a particular integral

$$
F_{p}=\sum_{i=2}^{k+2} \sum_{j=0}^{i} h_{j, i-j} x^{i} y^{j-i}
$$

of (2.7) can be found without difficulty. The Laurent expansions of $\omega_{k}\left(\zeta_{k}\right)$, along with (5.1) and the $\zeta_{k}$-plane transform ${ }^{16}$ of $\left.F_{p}\right]_{c_{0}}$, are now substituted in (4.9). The resulting equations yield recurrence formulas for the coefficients $\gamma_{n k}$ (or $\gamma_{n 1}$ and $\mu_{n 1}$ ).

For the case of the circular plate, the contour $C_{0}$ is a circle and the mapping functions are easily found to be

$$
z_{k}=\omega_{k}\left(\zeta_{k}\right)=a p_{k}\left(\zeta_{k}+\frac{\Gamma_{k}}{\zeta_{k}}\right), \quad \Gamma_{k}=\frac{\bar{q}_{k}}{p_{k}}, . \quad k=1,2 .
$$

Moreover, if $\omega_{k}\left(\zeta_{k}\right)$ are given by (5.4), then a consequence of the single-valuedness of $F_{k}\left(z_{k}\right)$ and $G_{1}\left(z_{1}\right)$ is that (4.7) may be written

$$
\phi_{k}\left(\zeta_{k}\right)=\sum_{n=0}^{\infty} \gamma_{n k}\left(\zeta_{k}^{n}+\frac{\Gamma_{k}^{n}}{\zeta_{k}^{n}}\right), \quad \psi_{1}\left(\zeta_{1}\right)=\sum_{n=0}^{\infty} \mu_{n 1}\left(\zeta_{1}^{n}+\frac{\Gamma_{1}^{n}}{\zeta_{1}^{n}}\right) .
$$

The direct mapping function corresponding to (5.4) is

$$
z=a s_{0}
$$

and hence it follows that

$$
\left.F_{p}\right]_{c_{0}}=\sum_{n=0}^{k+2}\left(C_{n} \sigma^{n}+\bar{C}_{n} \bar{\sigma}^{n}\right) \equiv C(\sigma) .
$$

For $k=0$ the operator (4.10) becomes $\sigma(\partial / \partial \sigma)+\bar{\sigma}(\partial / \partial \bar{\sigma})$, and one then obtains

$$
\left.i \sigma \frac{d s}{d \sigma} \frac{\partial F_{p}}{\partial n}\right]_{C_{0}}=\sum_{n=0}^{k+2}\left(D_{n} \sigma^{n}+\bar{D}_{n} \bar{\sigma}^{n}\right) \equiv D(\sigma) .
$$

The coefficients $C_{n}$ and $D_{n}$ are in general complex constants.

If $A(\sigma)$ and $B(\sigma)$ denote the right-hand members of (4.9a) and (4.9b) respectively, then the insertion of (5.1) and (5.6) in $A$ and $B$ yields

where

$$
A(\sigma)=\frac{a}{2} \sum_{n=0}^{k_{1}-1}\left(A_{n} \sigma^{n}+\bar{A}_{n} \bar{\sigma}^{n}\right), \quad B(\sigma)=\frac{a}{2} \sum_{n=0}^{k_{1}-1}\left(B_{n} \sigma^{n}+\bar{B}_{n} \bar{\sigma}^{n}\right)
$$

${ }^{16}$ This transform is most easily found by using the mapping function $z=\omega_{0}\left(\zeta_{0}\right)$, obtained by setting $k=0$ in (4.5), and defining $\mu_{0}=i$. In this way one achieves a direct mapping from the $z$ - to the 50 -plane, and maps any point of $C_{0}$ into a point $\sigma_{0}$ with the same coordinates as that given by the successive mappings (3.3) and (4.5). 


$$
\begin{aligned}
& A_{n}= \begin{cases}c_{1}, & n=0, \\
\bar{c}_{0}+c_{2}+c, & n=1, \\
c_{n+1}+\bar{c}_{-n+1}, & n=2,3, \cdots, k_{1}-1 ;\end{cases} \\
& B_{n}= \begin{cases}\sum_{j=-k_{1}}^{k_{1}} \frac{c_{j}+\bar{c}_{j}}{j-1}-c^{\prime}+\frac{1}{a} c^{\prime \prime \prime}, & n=0, \\
\bar{c}_{0}-c_{2}+\bar{c}, & n=1, \\
\frac{\bar{c}_{-n+1}-c_{n+1}}{n}, & n=2,3, \cdots, k_{1}-1\end{cases}
\end{aligned}
$$

and the prime on $\sum$ indicates that the term corresponding to $j=1$ is to be omitted. For convenience in writing, let

$$
\Pi(\sigma)=\sum_{n=0}^{k_{2}}\left(\Pi_{n} \sigma^{n}+\bar{\Pi}_{n} \bar{\sigma}^{n}\right), \quad \Lambda(\sigma)=\sum_{n=0}^{k_{2}}\left(\Lambda_{n} \sigma^{n}+\bar{\Lambda}_{n} \bar{\sigma}^{n}\right), \quad k_{2}=\max \left[k+2, k_{1}-1\right],
$$

where

$$
\Pi_{n}=A_{n}-D_{n}, \quad \Lambda_{n}=B_{n}-C_{n},
$$

with $A_{n}, B_{n}=0$ if $n \geqq k_{1}$, and $C_{n}, D_{n}=0$ if $n \geqq k+3$.

If $X_{n}^{0}$ and $Y_{n}^{0}$ are such that (5.1) is valid, then condition (4.4a) is satisfied, and (4.4b) demands that $c_{1}=\bar{c}_{1}$.

The determination of $\phi_{k}$ and $\psi_{1}$ is simplified by using not the boundary conditions (4.9), but an equivalent set. ${ }^{17}$ For the case of equal roots these are

$$
\begin{gathered}
\bar{\omega}(\bar{\sigma}) \phi(\sigma)+\omega(\sigma) \bar{\phi}(\bar{\sigma})+\psi(\sigma)+\psi(\bar{\sigma})=\Lambda(\sigma), \\
\bar{\omega}(\bar{\sigma}) \sigma \phi^{\prime}(\sigma)+\omega^{\prime}(\sigma) \sigma \bar{\phi}(\bar{\sigma})+\sigma \psi^{\prime}(\sigma) \\
=\frac{1}{2}\left\{\frac{p}{\bar{H}(\bar{\sigma})}-\frac{\bar{q}}{H(\sigma)}\right\}\left\{\Pi(\sigma) \bar{\sigma} \psi(\bar{\sigma})+\frac{1}{\beta} \sigma \Lambda^{\prime}(\sigma) \bar{\omega}^{\prime}(\bar{\sigma}) \bar{J}(\bar{\sigma})\right\} .
\end{gathered}
$$

Subscripts have been omitted in the above, since the distinction between the $z_{1}$ - and $z_{2}$-planes is not involved in the discussion. Substitution in (5.12) of the $\operatorname{series}^{18}(6.4)$ for $\phi(\sigma)$ and $\psi(\sigma)$, together with $\omega(\sigma)$ from (5.4), then yields recurrence relations for $\gamma_{n}$ and $\mu_{n}$. For the explicit form of these rather lengthy formulas, the reader is referred to the author's thesis. ${ }^{19}$

It should be noted that equations (5.12) are valid for any given boundary of the admissible class, provided the functions $\Pi(\sigma)$ and $\Lambda(\sigma)$ corresponding to this boundary are found.

The treatment of the case $\mu_{1} \neq \mu_{2}$ does not depart materially from that of the case of equal roots, and hence will be omitted here..$^{20}$

6. Extent of arbitrariness in solutions. Since a certain amount of arbitrariness is present in the functions occurring in the Muschelišvili solution of the plane stress problem for isotropic media, it is reasonable to ask if this phenomenon persists in the

\footnotetext{
${ }^{17}$ V. Morkovin, loc. cit.

${ }^{18}$ That $\phi$ and $\psi$ may be so written is clear after reading Section 6.

${ }^{19}$ Thermal deflection of anisotropic thin plates, University of Wisconsin, 1943.

${ }^{20}$ For details see the reference of footnote 19.
} 
case of anisotropic media. This question is answered affirmatively below, and the extent of the arbitrariness determined for the case of equal roots. This arbitrariness stems in part from the fact that the stress function is here, as in the Muschelišvili theory, the real part of an analytic function. In addition, it will be noted that the boundary conditions (5.12) contain three arbitrary constants, and one may expect this arbitrariness to be manifested in the solution.

If the prescribed functions $X_{n}^{0}(s)$ and $Y_{n}^{0}(s)$ satisfy the conditions (4.4) and are representable in the form (5.1), then the uniqueness theorem ${ }^{21}$ for the first boundary value problem of elasticity assures one that for a given distribution of temperature $T_{0}$, the state of stress in the interior of the plate is determinate. Since the stresses are given by (2.6), it is thus seen that the second derivatives of $F$ are determined in $S_{0}$.

If one lets $F_{h}$ be a solution of (2.7) and (4.3), lets $F_{h}^{(1)}=2 \operatorname{Re}\left\{\bar{z}_{1} F_{1}^{(1)}\left(z_{1}\right)+G_{1}^{(1)}\left(z_{1}\right)\right\}$ be another solution having the same second derivatives as $F_{h}$, and lets

$$
F_{h}^{(2)}=F_{h}-F_{h}^{(1)}=2 \operatorname{Re}\left\{\bar{z}_{1} F_{1}^{(2)}\left(z_{1}\right)+G_{1}^{(2}\left(z_{1}\right)\right\}
$$

then it follows that

$$
\frac{\partial^{2} F_{h}^{(2)}}{\partial x^{2}}=\frac{\partial^{2} F_{h}^{(2)}}{\partial x \partial y}=\frac{\partial^{2} F_{h}^{(2)}}{\partial y^{2}}=0
$$

throughout $S_{0}$. The real and imaginary parts of $F_{1}^{(2)}$ and $G_{1}^{(2)}$ must satisfy the CauchyRiemann equations in $S_{1}$, and these together with the three equations (6.2) enable one to show that

$$
\begin{aligned}
& F_{1}^{(2)}\left(z_{1}\right)=-i \eta_{3} z_{1} \dot{+}\left(\eta_{1}+i \eta_{2}\right), \\
& G_{1}^{(2)}\left(z_{1}\right)=\left(\nu_{3}+i \nu_{4}\right) z_{1}+\left(\nu_{1}+i \nu_{2}\right),
\end{aligned}
$$

where $\nu_{i}$ and $\eta_{i}$ are arbitrary real constants. Thus the functions (4.7) and

$$
\begin{aligned}
& \phi_{1}\left(\zeta_{1}\right)=\eta_{1}+i \eta_{2}-i \eta_{3} a p_{1}\left(\zeta_{1}+\frac{\Gamma_{1}}{\zeta_{1}}\right)+\phi_{1}^{(1)}\left(\zeta_{1}\right), \\
& \psi_{1}\left(\zeta_{1}\right)=\nu_{1}+i \nu_{2}+\left(\nu_{3}+i \nu_{4}\right) a p_{1}\left(\zeta_{1}+\frac{\Gamma_{1}}{\zeta_{1}}\right)+\psi_{1}^{(1)}\left(\zeta_{1}\right),
\end{aligned}
$$

describe the same state of stress in $S_{0}$. The arbitrariness in (6.3) is removed by choosing $\nu_{i}$ and $\eta_{i}$ so as to simplify $\phi_{1}$ and $\psi_{1}$. The choice made here is such that

$$
\phi_{1}\left(\zeta_{1}\right)=\sum_{n=1}^{\infty} \gamma_{n 1}\left(\zeta_{1}^{n}+\frac{\Gamma_{1}^{n}}{\zeta_{1}^{n}}\right), \quad \psi_{1}\left(\zeta_{1}\right)=\sum_{n=2}^{\infty} \mu_{n 1}\left(\zeta_{1}^{n}+\frac{\Gamma_{1}^{n}}{\zeta_{1}^{n}}\right) .
$$

where $\gamma_{11}=\bar{\gamma}_{11}$.

For the case $\mu_{1} \neq \mu_{2}$, a more lengthy consideration ${ }^{22}$ shows that one may write

$$
\phi_{1}\left(\zeta_{1}\right)=\sum_{n=2}^{\infty} \gamma_{n 1}\left(\zeta_{1}^{n}+\frac{\Gamma_{1}^{n}}{\zeta_{1}^{n}}\right), \quad \phi_{2}\left(\zeta_{2}\right)=\sum_{n=1}^{\infty} \gamma_{n 2}\left(\zeta_{2}^{n}+\frac{\Gamma_{2}^{n}}{\zeta_{2}^{n}}\right),
$$

where $\gamma_{22}=\bar{\gamma}_{22}$.

In both cases the arbitrariness is a reflection of that inherent in the values $f(s)$

${ }^{21}$ For details see the reference of footnote 19.

${ }^{22}$ See the reference of footnote 19. 
and $g(s)$ which $F$ must assume along $C_{0}$. The choice of $\nu_{i}$ and $\eta_{i}$ implied by (6.4) (or (6.5)) is found to dictate the selection of the arbitrary constants in (4.3), or in (4.9), the modified form of these boundary conditions, and conversely. This somewhat inverted method of eliminating the arbitrariness in $\phi_{1}$ and $\psi_{1}$ (or $\phi_{1}$ and $\phi_{2}$ ) is adopted in order to have these functions assume the form usual in the Muschelišvili theory. ${ }^{23}$

7. The differential equation for the deflection; the associated boundary conditions. The coefficients $N_{x x}, N_{y y}$, and $N_{x y}$ in (2.13) may now be regarded as known, and the thermo-elastic deflection problem for the anisotropic thin plate is reduced to that of solving the partial differential equation (2.13) for $w$, subject to the appropriate boundary conditions to be satisfied on $C_{0}$. For the first and second boundary value problems of plate theory these are, respectively,

$$
M_{n}=m(s), \quad Q_{n}+\frac{\partial H_{n s}}{\partial s}=p(s), \quad \text { on } \quad C_{0},
$$

and

$$
w=w(s), \quad \frac{\partial w}{\partial n}=w_{n}(s), \text { on } C_{0},
$$

where $m, p, w$, and $w_{n}$ are prescribed functions along $C_{0}$. The quantities ${ }^{24} M_{n}, H_{n}$ and $Q_{n}$ are the flexural couple, torsional couple, and the shearing force, respectively, which act on the edge of the plate.

The specialization of (2.7) and (2.13) to the isotropic case will now be given, since the resulting equations will be used in the sequel. For isotropic media

$$
\begin{aligned}
& a_{i i}=\frac{1}{E}, \quad i=1,2,3, \quad a_{i i}=\frac{2(1+\sigma)}{E}, \quad i=4,5,6, \\
& a_{i j}=-\frac{\sigma}{E}, \quad i, j=1,2,3, i \neq j, \quad a_{i j}=0, i, j=4,5,6, i \neq j, \\
& a_{i}=\alpha, i=1,2,3, \quad a_{i}=0, i=4,5,6,
\end{aligned}
$$

where $E$ denotes Young's modulus, $\sigma$ is Poisson's ratio, and $\alpha$ is the coefficient of thermal expansion. One easily finds that

$$
\begin{aligned}
b_{11}=b_{22} & =\frac{E}{1-\sigma^{2}}, & b_{66} & =\frac{E}{1+\sigma}, \\
b_{12} & =\frac{E \sigma}{1-\sigma^{2}}, & b_{16}=b_{26} & =0, \\
\alpha_{1}=\alpha_{2} & =\frac{\alpha E}{1-\sigma}, & \alpha_{6} & =0 .
\end{aligned}
$$

Using these values of the elastic parameters, one obtains from (2.7) and (2.13) the equations

$$
\begin{gathered}
\nabla^{4} F=-\alpha E \nabla^{2} T_{0}, \\
\nabla^{4} w=-\alpha(1+\sigma) \nabla^{2} T_{1}+\frac{1}{\dot{D}}\left\{N_{x x} \frac{\partial^{2} w}{\partial x^{2}}+2 N_{x y} \frac{\partial^{2} w}{\partial x \partial y}+N_{y y} \frac{\partial^{2} w}{\partial y^{2}}\right\},
\end{gathered}
$$

${ }^{23}$ I. S. Sokolnikoff, loc. cit., pp. 243-251.

${ }^{24}$ These are linear functions of $M_{x}, M_{y}$ and $H_{x y}$. See I. S. Sokolnikoff, loc. cit., p. 326. 
where $D=2 E h^{3} / 3\left(1-\sigma^{2}\right)$. Except for changes in notation, these are the same equations that Nádai ${ }^{25}$ obtains. The quantities (7.2) and (7.3) must also be inserted in (7.1) in order to obtain the boundary conditions for the isotropic plate.

A solution of the deflection equation (2.13) valid throughout the domain $S_{0}$ and satisfying the given boundary conditions on $C_{0}$, is not available; the same is true of (7.5) and its associated boundary conditions. ${ }^{26}$ By further specialization, however, it is possible to obtain the solution of the thermo-elastic problem for a case which is of some interest. This will be done in the following sections.

8. The istropic circular plate with radial temperature distribution. Let us consider an isotropic circular plate of radius $a$, and let it be subjected to a temperature distribution given by

$$
T(x, y, z)=T_{0}(r)+z T_{1}(r),
$$

where $r=\sqrt{x^{2}+y^{2}}$, and the origin is assumed to be at the center of the plate. As in section 2, the second derivatives of $T_{0}$ and $T_{1}$ are assumed continuous on $0 \leqq r \leqq a$. The edge of the plate is taken to be subjected to a uniform force $P$ per unit length of the arc parameter $s$.

It has been seen that before the deflection $w$ can be found, it is necessary to solve (7.4) for $F$. In the case at hand, $F_{p}$ can be found easily whether or not radial symmetry exists, for if $F$ is a solution of

$$
\nabla^{2} F=-\alpha E T_{0}
$$

then it is also a solution of (7.4). But (8.2) is the well-known Poisson's equation, and a solution is at once available from potential theory. Since $T_{0}$ has radial symmetry in the present case, however, the Laplacian operator becomes

$$
\nabla^{2}=\frac{1}{r} \frac{d}{d r}\left(r \frac{d}{d r}\right)
$$

and successive integrations of (8.2) give

$$
F_{p}(r)=-\alpha E \int_{0}^{r} \frac{d \xi}{\xi} \int_{0}^{\xi} T_{0}(\chi) \chi d \chi
$$

as the particular solution of (7.4) which is needed in $F$ (see (3.2)). For the isotropic plate, the roots of the characteristic equation (3.1) are $\mu_{1}=\mu_{2}=i$ and their conjugates. The transformation (4.5) becomes

$$
z=a \zeta
$$

and if $|\zeta|=\rho$, then $r=a \rho$. If one lets $f_{p}(\rho) \equiv F_{p}(a p)$, then from (5.7)

and

$$
C(\sigma)=f_{p}(\sqrt{\sigma \bar{\sigma}})=f_{p}(1),
$$

$$
\left.\left.D(\sigma)=i \sigma \frac{d s}{\partial \sigma} \frac{\partial F_{p}}{\partial n}\right]_{C_{0}}=\left(\sigma \frac{\partial}{\partial \sigma}+\bar{\sigma} \frac{\partial}{\partial \bar{\sigma}}\right) f_{p}(\rho)\right]_{\rho=1}=\sqrt{\sigma \bar{\sigma}} f_{p}^{\prime}(\sqrt{\sigma \bar{\sigma}})=f_{p}^{\prime}(1)
$$

${ }^{25}$ A. Nádai, loc. cit., pp. 264-268.

${ }^{26}$ Recent work by S. Bergman gives promise of extension to equations of the type (2.13) and (7.5). Bergman considers an equation a special case of which is

$\nabla^{4} u+a u_{x x}+2 b u_{x y}+c y_{y y}+d u_{x}+e u_{y}+f u=0$,

where $a, b, \cdots, f$ are analytic. functions of $x$ and $y$. See Duke Math. J. 11, 617-649 (1944). 
i.e., $C(\sigma)$ and $D(\sigma)$ reduce to constants on $\gamma$. This result is clearly a consequence of the fact that $T_{0}$ is a function of $r$ alone. One finds that

$$
\begin{aligned}
& C_{0}=\frac{1}{2} f_{p}(1), \quad C_{j}=0, \quad j=1,2, \cdots, \\
& D_{0}=\frac{1}{2} f_{p}^{\prime}(1), \quad D_{j}=0, \quad j=1,2, \cdots .
\end{aligned}
$$

With the edge forces as specified above, it follows that one may write

$$
X_{n}^{0}+i Y_{n}^{0}=-P e^{i \theta}
$$

and then (4.2) yields

$$
\left(\frac{\partial F}{\partial x}+i \frac{\partial F}{\partial y}\right)_{c_{0}}=a P(1-\sigma)+c .
$$

This is of the assumed form (5.1), and the constants defined in that expression have the values

$$
c_{0}=a P, \quad c_{1}=-a P .
$$

These are now inserted in (5.10) and the result together with (8.8) substituted in (5.11). Proceeding as indicated in section 5, one finds that the recurrence formulas simplify to

$$
\gamma_{1}=-\frac{a}{4}\left\{P+\frac{1}{a^{2}} f_{p}^{\prime}(1)\right\}, \quad \gamma_{n}=0, n=2,3, \cdots, \quad \mu_{n}=0, n=2,3, \cdots .
$$

The above results in conjunction with ( $3.2 \mathrm{~b})$ enable one to write

$$
F(r)=\frac{1}{2}\left\{-P+\frac{\alpha E}{r^{2}} \int_{0}^{r} T_{0}(\chi) \chi d \chi\right\} r^{2}-\alpha E \int_{0}^{r} \frac{d \xi}{\xi} \int_{0}^{\xi} T_{0}(\chi) \chi d \chi .
$$

In view of the radial symmetry, it is expedient to write (7.5) in terms of polar coordinates in the form

$$
\begin{aligned}
\nabla^{4} w=-\alpha(1+\sigma) \nabla^{2} T_{1}+\frac{1}{D}\left\{N_{r r} \frac{\partial^{2} w}{\partial r^{2}}+2 N_{r \theta}\right. & \frac{\partial}{\partial r}\left(\frac{1}{r} \frac{\partial w}{\partial \theta}\right) \\
& \left.+N_{\theta \theta}\left(\frac{1}{r} \frac{\partial w}{\partial r}+\frac{1}{r^{2}} \frac{\partial^{2} w}{\partial \theta^{2}}\right)\right\},
\end{aligned}
$$

where $N_{r r}=2 h \tau_{r r}^{0}, N_{r \theta}=2 h \tau_{r \theta}^{0}, N_{\theta \theta}=2 h \tau_{\theta \theta}^{0}$, and

$$
\tau_{r r}^{0}=\frac{1}{r} \frac{\partial F}{\partial r}+\frac{1}{r^{2}} \frac{\partial^{2} F}{\partial \theta^{2}}, \quad \tau r_{\theta}^{0}=-\frac{\partial}{\partial r}\left(\frac{1}{r} \frac{\partial F}{\partial \theta}\right), \quad \tau_{\theta \theta}^{0}=\frac{\partial^{2} F}{\partial r^{2}} .
$$

With $F$ of the form (8.11), these stresses become

$$
\begin{aligned}
\tau_{r r}^{0} & =-P+\frac{\alpha E}{a^{2}} \int_{0}^{a} T_{0}(\chi) \chi d \chi-\frac{\alpha E}{r^{2}} \int_{0}^{r} T_{0}(\chi) \chi d \chi, \\
\tau_{\theta \theta}^{0} & =-P+\frac{\alpha E}{a^{2}} \int_{0}^{a} T_{0}(\chi) \chi d \chi+\frac{\alpha E}{r^{2}} \int_{0}^{r} T_{0}(\chi) \chi d \chi-\alpha E T_{0}(r), \\
\tau_{r \theta}^{0} & =0 .
\end{aligned}
$$


Since $T_{1}, N_{r r}, N_{r \theta}$, and $N_{\theta \theta}$ are functions of $r$ alone, $\nabla^{2}$ has the form (8.3), and the equation for the deflection may therefore be written

$$
\begin{aligned}
\frac{d}{d r}\left(r \frac{d}{d r}\left[\frac{1}{r} \frac{d}{d r}\left\{r \frac{d w}{d r}\right\}\right]\right)= & -\alpha(1+\sigma) \frac{d}{d r}\left(r \frac{d T_{1}}{d r}\right) \\
+ & \frac{2 h}{D}\left[\left(P_{0} r-\frac{\alpha E}{r} \int_{0}^{r} T_{0}(\chi) \chi d \chi\right) \frac{d^{2} w}{d r^{2}}\right. \\
& \left.+\left(P_{0}+\frac{\alpha E}{r^{2}} \int_{0}^{r} T_{0}(\chi) \chi d \chi-\alpha E T_{0}\right) \frac{d w}{d r}\right],
\end{aligned}
$$

where $P_{0}$ is the constant

$$
P_{0}=\frac{\alpha E}{a^{2}} \int_{0}^{a} T_{0}(\chi) \chi d \chi-P .
$$

Equation (8.15) may be integrated with respect to $r$ and an equation obtained thereby which is not only of lower order than (8.15), but which also has simpler coefficients. Noting that

$$
\begin{aligned}
\frac{d}{d r}\left\{\left(P_{0} r\right.\right. & \left.\left.-\frac{\alpha E}{r} \int_{0}^{r} T_{0}(\chi) \chi d \chi\right) \frac{d w}{d r}\right\} \\
& =\left(P_{0} r-\frac{\alpha E}{r} \int_{0}^{r} T_{0}(\chi) \chi d \chi\right) \frac{d^{2} w}{d r^{2}}+\left(P_{0}+\frac{\alpha E}{r^{2}} \int_{0}^{r} T_{0}(\chi) \chi d \chi-\alpha E T_{0}\right) \frac{d w}{d r},
\end{aligned}
$$

we can carry out the desired integration immediately, obtaining

$$
\begin{aligned}
\frac{d}{d r}\left\{\frac{1}{r} \frac{d}{d r}\left(r \frac{d w}{d r}\right)\right\} & \\
& =-\alpha(1+\sigma) \frac{d T_{1}}{d r}+\frac{2 h}{D}\left(P_{0}-\frac{\alpha E}{r^{2}} \int_{0}^{r} T_{0}(\chi) \chi d \chi\right) \frac{d w}{d r}+\frac{k_{1}}{r},
\end{aligned}
$$

where $k_{1}$ is a constant of integration. Thus for the thin circular plate under uniform compression on its edge, and with $T_{0}(r)$ and $T_{1}(r)$ arbitrary save for certain conditions of continuity, the problem of finding the thermo-elastic deflection is reduced to that of solving the third order differential equation (8.17) with appropriate boundary conditions.

A repetition of the above integration is impossible for $T_{0}$ and $T_{1}$ of the general nature assumed above. Therefore, in order to complete the integration of the equation, $T_{0}$ and $T_{1}$ will be supposed to have the form ${ }^{27}$

$$
T_{0}(r)=\frac{D}{2 h \alpha E} \sum_{j=0}^{m} t_{0 j} r^{i}, \quad T_{1}(r)=-\frac{1}{\alpha(1+\sigma)} \sum_{j=0}^{n} t_{1, \gamma^{j}},
$$

where $t_{0 j}, t_{1 j}$ are real constants and $m, n$ are arbitrary positive integers. The solution of (8.17) may now be sought in the form of a power series. The polynomials $T_{0}$ and $T_{1}$ are now inserted in the differential equation, and if one makes the abbreviations

27 These polynomials may be regarded as approximations to power series representations of $T_{0}$ and $T_{1}$. 


$$
b_{j}=\frac{t_{0 j}}{j+2}, \quad d_{j}=j t_{1 j}
$$

the result is

$$
\frac{d^{8} w}{d r^{3}}+\frac{1}{r} \frac{d^{2} w}{d r^{2}}+\left\{\sum_{j=0}^{m} b_{j}\left(r^{j}-a^{j}\right)+\frac{2 h P}{D}-\frac{1}{r^{2}}\right\} \frac{d w}{d r}=\sum_{j=1}^{n} d_{j} r^{j-1} .
$$

The obvious replacement

$$
u(r)=\frac{d w}{d r}
$$

gives an equation of order two, and the further substitution

$$
b_{0}^{\prime}=\frac{2 h P}{D}-\sum_{j=1}^{m} b_{j} a^{j}
$$

then yields

$$
\frac{d^{2} u}{d r^{2}}+\frac{1}{r} \frac{d u}{d r}+\left(\sum_{j=1}^{m} b_{j} r^{j}+b_{0}^{\prime}-\frac{1}{r^{2}}\right) u=\sum_{j=0}^{n-1} d_{j+1} r^{j}
$$

as the equation to be solved for $u$. It will be observed that this equation has a regular singular point at $r=0$, and that the indices relative to this singular point are \pm 1 . Only the solution of the form

$$
u=r \sum_{i=0}^{\infty} \lambda_{i} r^{i}
$$

will be considered here. This represents the solution relative to $r=0$ which is bounded there. It is evident from physical considerations that this boundedness must obtain for the simply-connected plate, and hence it is sufficient to consider the solution of the above form. The series (8.22) is now inserted in (8.21) and the following recurrence relation defining the $\lambda_{j}$ is obtained:

$$
(j+4)(j+2) \lambda_{j+2}+b_{0}^{\prime} \lambda_{j}+\sum_{i=0}^{m} \lambda_{j-i} b_{j}=\left\{\begin{array}{cl}
d_{j+2}, & j=-1,0, \cdots, n-2, \\
0, & j=n-1, n, \ldots
\end{array}\right.
$$

where $\lambda_{j}=0$, if $j<0$. These equations permit $\lambda_{j}$ to be expressed in terms of the arbitrary quantity $\lambda_{0}$ and the known quantities $b_{0}^{\prime}, b_{i}$, and $d_{i}$. It is not expedient to give a formula for $\lambda_{j}$, since such an expression would be quite lengthy. The first several $\lambda_{j}$ may be computed with little trouble if $m$ and $n$ are not large, but the labor involved increases rapidly as these numbers become larger.

Up to now, the series defined by (8.23) is a formal solution of the differential equation (8.21). It is not difficult to show that this series converges for $0 \leqq r \leqq a$ and hence is actually a solution of the equation. For the discussion of the convergnece it suffices to consider the series

$$
\sum_{i=h}^{\infty} \lambda_{i} r^{i}
$$

where $h=m+n+2$. If one defines 


$$
\begin{aligned}
M & =\max \left\{1,\left|b_{0}^{\prime}\right|,\left|b_{1}\right|,\left|b_{2}\right|, \cdots,\left|b_{n}\right|\right\}, \\
K & =\max \left\{\left|\lambda_{0}\right|,\left|\lambda_{1}\right|, \cdots,\left|\lambda_{h-1}\right|\right\},
\end{aligned}
$$

and sets $E(\theta)=(h+\theta)(h+2+\theta)$, repeated use of the recurrence relation (8.23) with $j>n-2$ enables one to deduce that

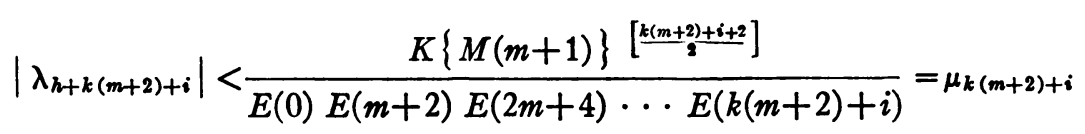

for $k=0,1,2, \cdots, i$ assuming the values $0,1, \cdots, m+1$ for each $k$. It may then be shown that the dominant series defined by the $\mu_{k(m+2)+i}$ converges uniformly for any finite $r$, and application of the well-known Weierstrass theorem yields the same convergence of (8.24) and (8.22). The desired solution $w$ of the deflection equation is then obtained by inserting (8.22) in (8.20) and integrating. We obtain

$$
w(r)=r^{2} \sum_{i=0}^{\infty} \kappa_{i} r^{i}+\kappa
$$

where $\kappa$ is a constant of integration and $\kappa_{i}=\lambda_{i} /(i+2)$. The uniform convergence of this integrated series on the interval $0 \leqq r \leqq a$ follows immediately from that of (8.22). Reference to the recursion formula (8.23) reveals that one may write

$$
\boldsymbol{\kappa}_{i}=\lambda_{0} \xi_{i}+\delta_{i}
$$

where $\xi_{i}$ contains the parameters $P, D, h, a$, and some or all of the $t_{0 j}$, while $\delta_{i}$ con. tains not only these but also some or all of the $t_{1 j}$. Thus the deflection may be written in the form

$$
w(r)=\lambda_{0} w_{0}(r)+w_{1}(r)+\kappa,
$$

where

$$
w_{0}(r)=\sum_{j=0}^{\infty} \xi_{j} r^{j}, \quad w_{1}(r)=\sum_{j=0}^{\infty} \delta_{j} r^{j}
$$

It is clear that $w_{1}(r)$ is a particular solution of $(8.21)$ and $w_{0}(r)$ the solution of the homogeneous equation which is bounded at $r=0$.

9. The circular plate with clamped and simply supported edge. The constant: $\lambda_{0}$ and $\kappa$ in (8.28) will be determined by the mode of support of the plate. The assump tion of radially symmetric deflection limits one to the consideration of boundary con ditions compatible with this type of deflection. The two methods of support mos commonly encountered are those of the clamped edge and the simply supported edge The boundary conditions for these are, respectively,

and

$$
\left.\left.\frac{d w}{d r}\right]_{r=a}=0, \quad w\right]_{r=a}=0,
$$

$$
\left.\left.M_{r}\right]_{r=a}=\left[\frac{d^{2} w}{d r^{2}}+\frac{\sigma}{r} \frac{d w}{d r}\right]_{r=a}=0, \quad w\right]_{r=a}=0 .
$$

The substitution of (8.28) in (9.1) and (9.2) yields for the plate with clamped edge the constants 


$$
\lambda_{0}=-\frac{a w_{1}^{\prime \prime}(a)+\sigma w_{1}^{\prime}(a)}{a w_{0}^{\prime \prime}(a)+\sigma w_{0}^{\prime}(a)}, \quad \kappa=\frac{a w_{1}^{\prime \prime}(a)+\sigma w_{1}^{\prime}(a)}{a w_{0}^{\prime \prime}(a)+\sigma w_{0}^{\prime}(a)} w_{0}(a)-w_{1}(a),
$$

and for the plate with a simply supported edge,

$$
\lambda_{0}=-\frac{w_{1}^{\prime}(a)}{w_{0}^{\prime}(a)}, \quad \kappa=\frac{w_{1}^{\prime}(a)}{w_{0}^{\prime}(a)} w_{0}(a)-w_{1}(a) .
$$

These quantities are now inserted in (8.28), giving for the plate with clamped edge the deflection

$$
w(r)=\frac{w_{1}^{\prime}(a)}{w_{0}^{\prime}(a)}\left\{w_{0}(a)-w_{0}(r)\right\}+w_{1}(r)-w_{1}(a),
$$

and for the plate with simply supported edge

$$
w(r)=\frac{a w_{1}^{\prime \prime}(a)+\sigma w_{1}^{\prime}(a)}{a w_{0}^{\prime \prime}(a)+\sigma w_{0}^{\prime}(a)}\left\{w_{0}(a)-w_{0}(r)\right\}+w_{1}(r)-w_{1}(a) .
$$

It will be assumed that $w_{0}^{\prime}(a)$ and $a w_{0}^{\prime \prime}(a)+\sigma w_{0}^{\prime}(a)$ do not vanish. It may be shown that an $a$ which causes the former (latter) to vanish is the radius of the clamped (simply supported) plate with given temperature distribution $T_{0}(r)$ for which $P$ is a critical (i.e., buckling) load. The question of stability is not under consideration here, hence the above assumption is made.

The case $m=0$ is of particular interest, for then $w$ contains the Bessel function of the first kind of order zero. In this case $T_{0}(r)$ reduces to a constant, and (8.21) becomes

$$
\frac{d^{2} u}{d r^{2}}+\frac{1}{r} \frac{d u}{d r}+\left(\frac{2 h P}{D}-\frac{1}{r^{2}}\right) u=\sum_{j=0}^{n-1} d_{j+1^{r}} .
$$

If the recurrence relation is written in terms of the $\kappa_{i}$ rather than the $\lambda_{i}$, the result is

$$
(i+4)^{2} \kappa_{i+2}+\frac{2 h P}{D} \kappa_{i}=\left\{\begin{array}{cl}
t_{1, i+2}, & i=-1,0, \cdots, n-2, \\
0 & i=n-1, n, \cdots,
\end{array}\right.
$$

from which one obtains easily the deflection

$$
w(r)=\lambda_{0} J_{0}\left(r \sqrt{\frac{2 h P}{D}}\right)+w_{10}(r)+\kappa .
$$

Here $w_{10}(r)$ designates the form which $w_{1}(r)$ assumes for $m=0$. Proceeding as in the more general case, for the plate with clamped edge we find that the deflection is

$$
\begin{aligned}
w(r)= & \frac{w_{10}^{\prime}(a)}{\sqrt{\frac{2 h P}{D}} J_{0}\left(a \sqrt{\frac{2 h P}{D}}\right)}\left\{J_{0}\left(r \sqrt{\frac{2 h P}{D}}\right)-J_{0}\left(a \sqrt{\frac{2 h P}{D}}\right)\right\} \\
& +w_{10}(r)-w_{10}(a),
\end{aligned}
$$

and for the plate with simply supported edge, 


$$
\begin{aligned}
w(r)=\frac{a w_{1}^{\prime \prime}(a)+\sigma w_{1}^{\prime}(a)}{\sqrt{\frac{2 h P}{D}}\left\{\sqrt{\frac{2 h P}{D}} J_{0}\left(a \sqrt{\frac{2 h P}{D}}\right)-(1-\sigma) J_{1}\left(a \sqrt{\frac{2 h P}{D}}\right)\right\}} \\
\cdot\left\{J_{0}\left(r \sqrt{\frac{2 h P}{D}}\right)-J_{0}\left(a \sqrt{\frac{2 h P}{D}}\right)\right\}+w_{10}(r)-w_{10}(a) .
\end{aligned}
$$

10. Conclusion. The problem of flexure is considered for a thin anisotropic elastic plate, subjected in its interior to a temperature distribution of the form

$$
T(x, y, z)=T_{0}(x, y)+z T_{1}(x, y) .
$$

The usual assumptions of thin plate theory, together with Hooke's law extended to encompass thermal effects, permit one to derive two partial differential equations governing the deflection of the plate. For the isotropic plate these equations specialize to those given by Nádai. If thermal effects are supposed absent, and $N_{x x}, N_{y y}, N_{x y}$ are interpreted as arising from edge forces alone, then (2.13) becomes the equation for the deflection of an anisotropic thin plate stressed in its own plane.

A method is given for determining the stress function $F$ for a plate with edge forces representable in the form of a trigonometric polynomial, and the determination of $F$ is carried out for the circular plate.

For the thermo-elastic problem formulated with the above generality, a suitable solution of the deflection equation is not available. Accordingly, the problem is specialized to the simpler case of the isotropic circular plate with radially symmetric temperature distribution and $T_{0}(r), T_{1}(r)$ in the form of polynomials. The solution of the resulting deflection equation may be found in the form of a power series, and convergence established. Boundary conditions for the clamped and simply supported plate are then considered, and the deflection for each of these modes of support determined. 